use ionising radiation. Here we present the first single-breath demonstration of collateral ventilation, by using hyperpolarised gas MRI.

Methods Ten patients with moderate to severe COPD as defined by NICE guidelines were scanned using a 1.5T whole body MRI system. A mix of $200 \mathrm{ml}$ hyperpolarised ${ }^{3} \mathrm{He}$ and $800 \mathrm{ml} \mathrm{N}_{2}$ was inhaled, and ${ }^{3} \mathrm{He} \mathrm{MR}$ images were acquired. $3 \mathrm{D}$ images with full lung coverage were acquired at six time-points during a single breathhold.

Results Abstract S118 figure 1 shows example sequential images in one patient, with insets highlighting an area with late ventilation. Hyperpolarised ${ }^{3} \mathrm{He} \mathrm{MR}$ signal is non-renewable and diminishes over time. In normally ventilated regions of the lung this expected signal decay is observed. However, in the regions indicated with arrows the signal increases over time due to collateral ventilation, with a progressive influx of polarised gas from the edge of the defects towards the centre. The long time constants for ventilation of some areas are not compatible with their ventilation via their feeding bronchi. Instances of collateral ventilation were observed in eight of the ten patients scanned. These examples varied in defect size, number and fill-rate from the strongest case (Abstract S118 figure 1) to much more subtle effects.

Conclusions A method for direct visualisation of collateral ventilation within a single breath-hold has been demonstrated in COPD patients for the first time. The technique gives $3 \mathrm{D}$ full lung coverage, and is non-invasive and non-ionising. The ability to image collateral ventilation directly may help to understand pathophysiology in COPD and aid assessment for therapy.

\section{S119 UK HOT-HMV TRIAL: ACCEPTABILITY AND TOLERABILITY OF HIGH PRESSURE DOMICILIARY NON-INVASIVE VENTILATION (NIV) IN COPD}

doi:10.1136/thoraxjnl-2011-201054b.119

${ }^{1} \mathrm{P}$ B Murphy, ${ }^{1} \mathrm{~J}$ Moxham, ${ }^{2} \mathrm{M}$ I Polkey, ${ }^{3} \mathrm{~N}$ Hart. ${ }^{1}$ King's College London, London, UK; ${ }^{2}$ NIHR, Respiratory Biomedical Research Unit, Royal Brompton Hospital and Imperial College London, London, UK; ${ }^{3}$ Guy's \& St Thomas' NHS Foundation Trust and Kings College London NIHR Biomedical Research Centre, London, UK

Introduction Domiciliary NIV in COPD remains controversial with previous randomised controlled trials showing little clinical benefit. However, these trials have been criticised for using low pressure NIV and consequently nocturnal hypoventilation occurs. In contrast, there are genuine concerns that patients may not be able to tolerate the high pressure domiciliary NIV required to manage nocturnal hypoventilation effectively.

Method Patients admitted for acute hypercapnic respiratory failure due to an exacerbation of COPD with persistent hypercapnia $\left(\mathrm{PaCO}_{2}>7 \mathrm{kPa}\right) 2-4$ weeks following resolution of acute episode were offered participation into the trial. Patients were randomised to either home oxygen therapy (HOT) or home mechanical ventilation

Abstract S119 Table 1 Baseline charateristics

\begin{tabular}{|c|c|c|}
\hline & HOT $(n=10)$ & HMV $(n=10)$ \\
\hline Age (years) & $68 \pm 9$ & $70 \pm 10$ \\
\hline $\mathrm{BMI}\left(\mathrm{kgm}^{-2}\right)$ & $26 \pm 6$ & $21 \pm 3$ \\
\hline $\mathrm{FEV}_{1}(\mathrm{I}) /(\%)$ & $0.73 \pm 0.34 / 31 \pm 7$ & $0.56 \pm 0.16 / 22 \pm 12$ \\
\hline FVC (I)/(\%) & $1.79 \pm 0.56 / 65 \pm 12$ & $1.72 \pm 0.64 / 48 \pm 18$ \\
\hline $\mathrm{PaCO}_{2}(\mathrm{kPa})$ & $7.94 \pm 0.73$ & $8.22 \pm 0.71$ \\
\hline $\mathrm{PaO}_{2}(\mathrm{kPa})$ & $6.88 \pm 1.01$ & $6.54 \pm 0.81$ \\
\hline $\mathrm{HCO}_{3}(\mathrm{mmol} / \mathrm{l})$ & $35 \pm 3$ & $37 \pm 4$ \\
\hline MRC (/5) & $4 \pm 1$ & $5 \pm 1$ \\
\hline SGRO (/100) & $63 \pm 12$ & $66 \pm 14$ \\
\hline SRI-SS (/100) & $49 \pm 14$ & $52 \pm 16$ \\
\hline $\mathrm{O}_{2}$ prescription $(\mathrm{L} / \mathrm{min})$ & $1.2 \pm 0.7$ & $1.0 \pm 0.4$ \\
\hline
\end{tabular}

(HMV) and followed up at 6 weeks and 3 months. Patient assessment included anthropometrics, arterial blood gases and health related quality of life measures. Sleep disruption was assessed using actigraphy (Actiwatch spectrum, Philips-Respironics, Murrysville, Pennsylvania, USA) for 7 days following the assessment.

Results 36 patients have been recruited and randomised to date Abstract S119 table 1 show the baseline data for 20 patients that have completed follow-up to 3 months. Discharge ventilator settings were IPAP $26 \pm 3 \mathrm{cmH}_{2} \mathrm{O}$, EPAP $5 \pm 1 \mathrm{cmH}_{2} \mathrm{O}$ and back up rate $15 \pm 1 \mathrm{bpm}$ in the $\mathrm{HMV}$ group. Total sleep time during the first 2 weeks of HMV was significantly shorter than in those patients receiving HOT (?88 $\mathrm{min}$; 95\% CI 5 to $172 \mathrm{~min}, \mathrm{p}=0.04$ ). However, by 6 weeks there was no difference between the groups ( $\Delta 66 \mathrm{~min}$; $95 \% \mathrm{CI}-62$ to $194 \mathrm{~min}, \mathrm{p}=0.3)$. This was sustained at 3 months ( $\Delta 51 \mathrm{~min} ; 95 \% \mathrm{CI}-100$ to $202 \mathrm{~min}, \mathrm{p}=0.5$ ). There were no between group differences in wake after sleep onset, sleep efficiency or sleep latency at initial assessment or subsequent follow-up. Ventilator compliance at 6 weeks was $3 \mathrm{~h} 41 \mathrm{~min} \pm 1 \mathrm{~h} 41 \mathrm{~min}$ and at 3 months was $4 \mathrm{~h} 30 \mathrm{~min} \pm 1 \mathrm{~h} 44 \mathrm{~min}$.

Conclusion A reduction in total sleep time between patients receiving HMV compared with those receiving HOT was demonstrated during the initial period of acclimatisation to HMV. However, despite increasing ventilator usage during the follow-up period the difference in sleep duration between treatment groups reduced suggesting improved tolerability to NIV.

\section{S120 REFERENCE VALUES FOR THE INCREMENTAL SHUTTLE WALKING TEST IN A HEALTHY POPULATION}

doi:10.1136/thoraxjnl-2011-201054b.120

S L Harrison, N J Greening, L Houchen, J E A Williams, M Morgan, M Steiner, S J Singh. Pulmonary Rehabilitation Research Group, Glenfield Hospital, UHL NHS Trust, Leicester, UK

Introduction The assessment of functional exercise capacity is important in the evaluation of patients with chronic conditions and field walking tests are often used to assess functional exercise

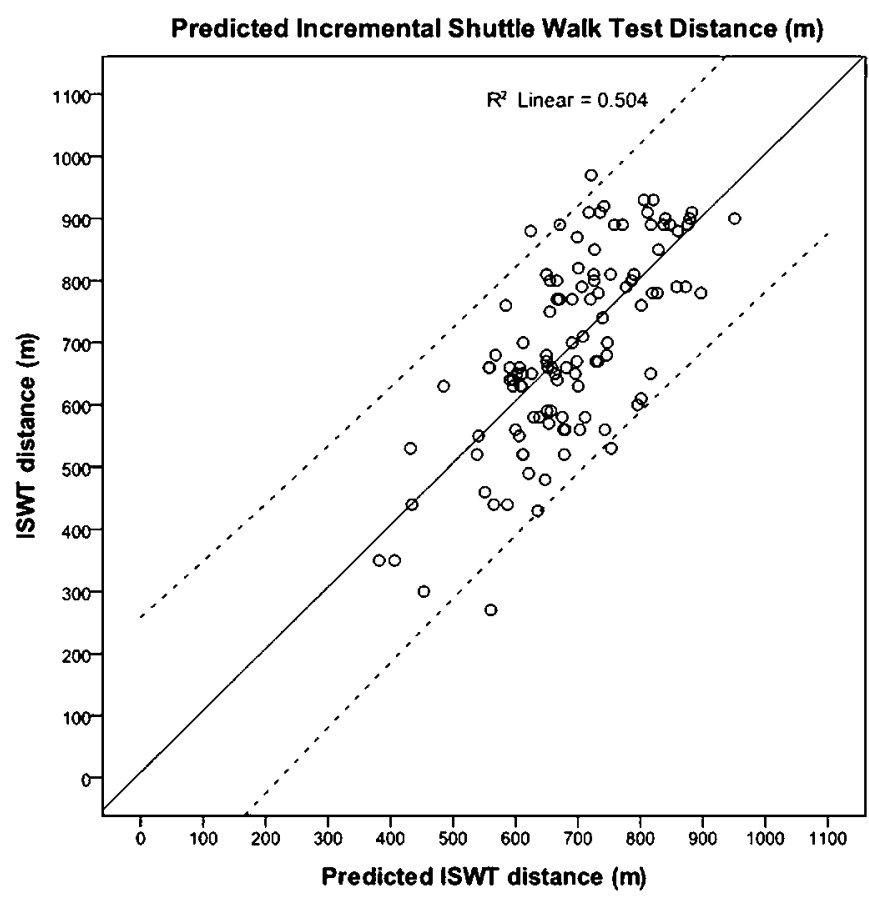

Abstract S120 Figure $1 \quad$ ISWTpred $=603.345+\left(61.870 \times \mathrm{FEV}_{1}\right)+$ $(6.960 \times 0 M V C)+(9.183 \times$ DUKE $)-(13.140 \times B M I)-(4.010 \times$ age $)$. 
capacity. The ISWT is one field exercise test which is often employed in the assessment of pulmonary and cardiac rehabilitation (PR, CR) patients and also to prescribe a walking speed. In PR and CR programmes the ISWT is employed to calculate walking speed set at a desired training threshold. The aim of the study was to establish reference values for the ISWT and an equation for its prediction in a healthy population. This will allow comparison between patients from $\mathrm{PR}$ and $\mathrm{CR}$ programmes and healthy age-matched controls.

Methods Subjects were aged between 40 and 90 years, had normal spirometry defined as $\mathrm{FEV}_{1} \%$ pred $>80 \%$ and/or a $\mathrm{FEV}_{1} / \mathrm{FVC}>70 \%$ and had no known co-morbidities affecting mobility. The best distance from two ISWT was recorded along with body mass index (BMI) and leg length. Quadriceps maximal voluntary contraction (QMVC: Kg) was measured using a strain gauge (Kern). Physical activity was assessed using the DUKE physical activity questionnaire and an activity monitor (SenseWear $\mathrm{PRO}^{2}$ Armband). The number of steps and energy expenditure achieved over 2 days was recorded. Subjects also completed the Hospital Anxiety and Depression Scale.

Results 114 patients completed the study [mean (SD) age 60.48 (10.99) years, $\mathrm{FEV}_{1} 108.82 \%$ (15.13) predicted, 37 male]. Mean ISWT distance was $690 \mathrm{~m}$ (152.68). There were no significant differences in walking distance between males and females ( $p>0.05)$. ISWT distance showed significant correlations with age, BMI, FEV 1 , OMVC, DUKE physical activity score and height $(p<0.01)$. Stepwise multiple regression analysis showed that age, $\mathrm{BMI}, \mathrm{FEV}_{1}, \mathrm{OMVC}$ and DUKE physical activity score were independent contributors to the ISWT distance achieved by healthy subjects, explaining $50.4 \%$ of the variance. (Abstract S120 figure 1). Conclusions Variance in the ISWT can be measured using a composite score, comprising of: age, BMI, $\mathrm{FEV}_{1}, \mathrm{OMVC}$ and DUKE physical activity score. These findings would allow clinicians to express results of the ISWT as a percentage of the predicted values making results more meaningful for patients with chronic conditions.

\section{Mast cells, smooth muscle and inflammation in asthma}

\section{S121 MEDIATOR PROFILING OF SEVERE ASTHMA PHENOTYPES}

doi:10.1136/thoraxjnl-2011-201054b.121

${ }^{1} \mathrm{D}$ Desai, ${ }^{1} \mathrm{C} J$ Newby, ${ }^{1} \mathrm{P}$ Haldar, ${ }^{1} \mathrm{~S}$ Shah, ${ }^{1} \mathrm{~S}$ Gupta, ${ }^{1} \mathrm{M}$ Bafadhel, ${ }^{1} \mathrm{~A}$ Singapuri, ${ }^{1} \mathrm{~S}$ Siddiqui, ${ }^{1} \mathrm{~J}$ Woods, ${ }^{2} \mathrm{~A}$ Herath, ${ }^{2} \mathrm{~K}$ Anderson, ${ }^{1} \mathrm{P}$ Bradding, ${ }^{1} \mathrm{R} \mathrm{H}$ Green, ${ }^{1} \mathrm{~A} J$ Wardlaw, ${ }^{1} \mathrm{D}$ D Pavord, ${ }^{2} \mathrm{R}$ D May, ${ }^{1} \mathrm{C}$ E Brightling. ${ }^{1}$ Institute for Lung Health, Department of Infection, Immunity \& Inflammation, University of Leicester, Leicester, UK; ${ }^{2}$ Medimmune Ltd, Milstein Building, Granta Park, Cambridge, UK

Background Severe asthma is a heterogeneous disease. Defining its phenotypic heterogeneity is likely to shed light upon its immunopathogenesis and direct therapy. We sought to determine the relationship between phenotypes of severe asthma and sputum mediator profiles.

Methods Subjects were recruited from a Difficult Asthma Clinic at a single centre $(n=164)$ and assessments of lung function, atopic status, asthma control and sputum induction were undertaken. Sputum was obtained and supernatants were analysed for 23 mediators using the Meso-Scale Discovery platform. We performed k-means cluster analysis to determine clinical clusters using the baseline characteristics and sputum differential counts. The pattern of mediator expression was determined by factor analysis to identify biological factors. The biological factors were related to the clinical clusters and subjects stratified by asthma control, exacerbation frequency, treatment and sputum cell counts. The repeatability of the individual clinical characteristics and biological mediators was assessed in paired samples in 106 subjects and in three samples in 66 subjects.

Results We identified four clinical clusters and five biological factors The biological factors were differentially expressed in subjects stratified by sputum cell counts, asthma control and exacerbation frequency, but were not significantly different across the clinical clusters. The within subject repeatability of mediators was moderate; biological factors were consistent and tracked with sputum cell counts for the repeated visits.

Conclusions Sputum mediator profiling of severe asthma revealed repeatable biological factors that were strongly associated with cellular profiles and inform our understanding of asthma phenotypes.

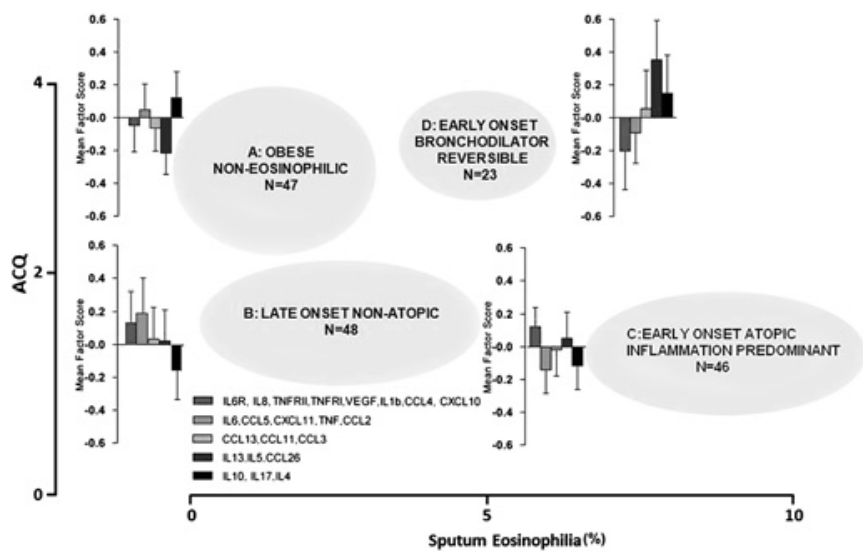

Abstract S121 Figure 1 Relationship between clinical clusters and biological factors. The clinical clusters are plotted in two dimensions with airway inflammation on the $x$ axis and asthma control (ACO) on the $y$ axis. The size of the ellipsoid represents the number of subjects within each clinical cluster. The distribution of the five biological factors for each cluster is shown as the mean (SEM) factor scores.

\section{S122 SPUTUM CYTOKINE PROFILES IN ASTHMA AND THE IMPACT OF SMOKING-A FACTOR ANALYSIS}

doi:10.1136/thoraxjnl-2011-201054b.122

${ }^{1} \mathrm{M}$ Spears, ${ }^{2} \mathrm{C}$ Dewet, ${ }^{1} \mathrm{C}$ McSharry, ${ }^{1} \mathrm{R}$ Chaudhuri, ${ }^{1} \mathrm{I}$ Donnelly, ${ }^{1} \mathrm{~L}$ Jolly, ${ }^{1} \mathrm{E}$ Cameron, ${ }^{1} \mathrm{~N}$ C Thomson. ${ }^{1}$ Institute of Infection, Immunity \& Inflammation, University of Glasgow, Glasgow, UK; ${ }^{2}$ NHS Education for Scotland, Glasgow, UK

Introduction Cigarette smokers with asthma have a distinct clinical phenotype from non-smokers with asthma. This may reflect altered airway inflammation although how cigarette smoking directs this is unclear. We employed exploratory factor analysis to examine the impact of smoking on airway inflammation.

Abstract S122 Table 1 Factor loadings

\begin{tabular}{llll}
\hline Rotated component matrix & & \\
\hline & Factor & $\mathbf{2}$ & $\mathbf{3}$ \\
\cline { 2 - 4 } & $\mathbf{1}$ & & \\
\hline IFN-? & 0.991 & & \\
IL-4 & 0.986 & & \\
IL-5 & 0.986 & 0.864 & \\
IL-6 & & 0.964 & 0.813 \\
CXCL9 & & 0.908 & 0.889 \\
CXCL10 & & 0.920 \\
CCL2 & & & \\
CCL3 & & & \\
CCL4 & & &
\end{tabular}

